\title{
NEURITE OUTGROWTH IN MOLLUSCAN ORGAN AND CELL CULTURES: THE ROLE OF CONDITIONING FACTOR(S) ${ }^{1}$
}

\author{
RICHARD G. WONG, ROBERT D. HADLEY, S. B. KATER, ${ }^{2}$ aND GARRY C. HAUSER \\ Department of Zoology, University of Iowa, Iowa City, Iowa 52242
}

\begin{abstract}
Isolated neurons from adult central ganglia of the snail Helisoma were cultured in vitro in modified Liebowitz L-15 medium. Such neurons displayed electrical excitability comparable to that in acutely dissected ganglia. Isolated neurons remained spherical in defined medium throughout culture durations up to 2 weeks. This static morphology was contrasted by the significant neuritic outgrowth which occurred from neurons maintained in medium with co-cultured intact Helisoma brains or in brain conditioned medium. A morphological sequence of growth cone formation and neurite extension occurred only in the presence of a conditioning factor(s) with a mode of action which included tight binding of the conditioning factor to the substratum. Under these conditions, the two primary neuronal phenotypes, electrical excitability and complex neuronal architecture, could be affected independently in adult molluscan neurons cultured in vitro.
\end{abstract}

Adult molluscan neurons possess a great capacity for neurite outgrowth, regeneration, and highly specific formation and re-formation of neuronal connections. Identified neurons of the snail, Helisoma, rapidly produce neurite outgrowth in response to damage and reliably regenerate to re-form specific connections (Murphy and Kater, 1978, 1980a). Chemical and electrical connections between central neurons also are modified transiently in response to peripheral axotomy (Bulloch et al., 1980). The changes which occur within the central ganglia in response to peripheral injury mimic the effects of axotomy of motor neurons described in mammalian spinal cord (e.g., Eccles et al., 1958). Additionally, during central growth, identified neurons of Helisoma can form novel electrical connections which are preceded by transient "inappropriate" cunnections. In this system, the breaking of inappropriate connections depends upon the formation of a stable, "correct" connection (Bulloch and Kater, 1981). The plasticity exhibited by adult molluscan neurons appears to be a composite, ranging from random outgrowth to preprogrammed selection of specific connections.

In vitro organ and cell culture methods offer clear advantages for the study of mechanisms underlying neu-

\footnotetext{
' We thank Joanne Kater, Gerald Sedgewick, and Paul Gade for photography and assistance in preparation of the manuscript. We gratefully acknowledge Drs. D. Barker, A. Bulloch, C. Cohan, J. Denburg, P. Letourneau, J. Nicholls, D. Ready, and C. -F. Wu for their instructive comments during the course of this investigation. This work was supported by Public Health Service Grants NS 15350 and NS 09696 and a grant from the Whitehall Foundation. Robert D. Hadley was supported by Training Grant MH 15172.

2 To whom correspondence should be addressed.
}

ronal growth and formation of connections by allowing ongoing observations and manipulations. All previous experiments on plasticity of adult molluscan neurons required experimental manipulation and subsequent culture of the ganglia. In order to provide conditions as nearly normal as possible, we employed an in vivo technique. Whole ganglia and effector systems from one animal were manipulated and subsequently implanted into the hemocoel of a host snail (Murphy and Kater, $1978,1980 \mathrm{a})$. This method insured that neurons were bathed by snail hemolymph throughout their periods of growth and formation of connections. The disadvantage of this method was that ongoing manipulations and observations of identified neurons were precluded by the fact that they were incubating within a host snail. The present investigation describes the behavior of Helisoma neurons cultured in vitro where precise observations and manipulations are routinely feasible.

The advantages of neuronal cell culture which have long been exploited in vertebrate neuronal culture systems are exemplified by the precision of investigations on the regulation of transmitter synthesis in mammalian sympathetic neurons (Reichart and Patterson, 1977; Furshpan et al., 1976). The culture of invertebrate neurons is in its relative infancy. Organ culture of some invertebrate ganglia has been used to study the stability of neuronal pacemakers in Aplysia (Beiswanger and Jacklet, 1975) and neuronal sprouting and regeneration in leeches (e.g., Nicholls et al., 1977). Cell isolation procedures have made it possible to culture isolated bag cells of Aplysia and to examine their physiological and biochemical properties (Kaczmarek et al., 1979). Ready and Nicholls (1979) have shown that identified neurons of the 
leech, when isolated, are capable of making connections only with a circumscribed set of other identified neurons. This combination of the ability to control environmental conditions precisely and to perform long term observations and manipulations on experimentally tractable neurons can be employed to study, at the cellular level, mechanisms underlying the formation of specific connections between identified neurons.

This study is an investigation into basic aspects of the growth of molluscan neurons cultured in vitro and is an extension of our previous work on in vitro organ culture (Hadley and Kater, 1979; Kater and Hadley, 1980; R. D. Hadley, R. G. Wong, S. B. Kater, and D. L. Barker, manuscript in preparation). We report here the effects of a conditioning factor (CF) derived from whole Helisoma brains that enhances neurite outgrowth from Helisoma neurons in ganglionic organ culture and is required for outgrowth from isolated neurons in culture. By examining CF and its effects on both electrical excitability and neurite extension, we conclude that CF acts as a substrate conditioning factor and affects primarily neurite outgrowth with no apparent effect on the maintenance of electrical excitability.

\section{Materials and Methods}

\section{Animals and dissection}

Animals were obtained from our inbred laboratory stock of Helisoma trivolvis $\left(\operatorname{Red}_{1}\right)$ which were maintained at 22 to $25^{\circ} \mathrm{C}$. Dissection procedures were as described by Kater and Kaneko (1972). Deshelled snails were placed first in 25\% Listerine (Warner-Lambert Pharmaceutical Co.) in Helisoma saline solution for 15 min and then transferred to antibiotic saline solution for 20 to $30 \mathrm{~min}$ before sterile dissection. Buccal ganglia and all central ganglia were removed under sterile conditions and prepared for organ or isolated cell culture. All sterile dissections were carried out in a Baker Edgegard laminar flow hood in sterile antibiotic saline.

\section{Culture media}

The composition of normal Helisoma saline (NS) was: $\mathrm{NaCl}, 51.3 \mathrm{~mm}$; KCl, $1.7 \mathrm{~mm} ; \mathrm{CaCl}_{2}, 4.1 \mathrm{~mm} ; \mathrm{MgCl}_{2}, 1.5$ mM; 5 mM HEPES (4-(2-hydroxyethyl)-1-piperazine-ethanesulfonic acid, $\mathrm{pH}$ 7.3). Sterile antibiotic fortified saline (ABS) additionally contained $500 \mathrm{units} / \mathrm{ml}$ of penicillin, $0.5 \mathrm{mg} / \mathrm{ml}$ of streptomycin, and $1.25 \mu \mathrm{g} / \mathrm{ml}$ of Fungizone. Serum-free Helisoma L-15 (HL-15) medium was made up of half-strength salt-free Liebowitz L-15 (Gibco, special order) with added inorganic salts and $100 \mathrm{units} / \mathrm{ml}$ of penicillin, $0.1 \mathrm{mg} / \mathrm{ml}$ of streptomycin, and $0.25 \mu \mathrm{g} / \mathrm{ml}$ of Fungizone. For culture media, inorganic salts and buffer were as for normal Helisoma saline except the $\mathrm{NaCl}$ was lowered to $40 \mathrm{~mm}$ to accommodate the osmotic contribution made by the addition of salt-free $L-15$. Two modifications of HL-15 were employed for ganglion culture and isolated neuron culture. Co-culture medium (CCHL-15) was prepared by adding intact dissected $\mathrm{Hel}$ isoma brains (two brains per ml) to normal HL-15 media. Conditioned medium (CMHL-15) was prepared from HL-15 as follows: dissected brains (two per $\mathrm{ml}$ ) were placed in normal HL-15 for $72 \mathrm{hr}$. Then the CMHL-15 was either passed through a $0.2-\mu \mathrm{m}$ Gelman or $0.22-\mu \mathrm{m}$
Millipore filter or used unfiltered. Cultures were maintained at 22 to $25^{\circ} \mathrm{C}$ in air in a humidified cabinet.

\section{Tissue preparation}

Organ culture of buccal ganglia is described elsewhere (Hadley and Kater, 1979; Kater and Hadley, 1980; R. D. Hadley, R. G. Wong, S. B. Kater, and D. L. Barker, manuscript in preparation). Buccal ganglia were secured to the bottom of polylysine-coated culture dishes by placing over them a drop of $0.6 \%$ Difco Bacto agar, $0.6 \%$ Knox unflavored gelatin in NS. The dishes then were flooded with culture medium. For single cell dissociation, each central ganglionic ring (consisting of visceral, parietal, pedal, and cerebral ganglia) was pinned down on a Sylgard (Dow Corning) pad by the cerebral ganglia and cut nerve trunks, and the whole ganglionic ring was treated with $0.5 \%$ pronase at $23^{\circ} \mathrm{C}$ in sterile NS for $2 \mathrm{~min}$. Then this was rinsed several times with sterile ABS and the connective tissue sheath covering the visceral, left parietal, and both paired pedal ganglia was cut with tungsten microknives, allowing the neuronal cell bodies to pop out of each ganglion. The desheathed ganglia were dissected out individually, dissociated by incubation at $23^{\circ} \mathrm{C}$ for 1 to $1.5 \mathrm{hr}$ in $0.1 \%$ trypsin (Sigma III) in HL-15, and then rinsed for $15 \mathrm{~min}$ in $0.1 \%$ trypsin inhibitor (Sigma I-S) in HL-15. The ganglia then were transferred to culture medium and dissociated into single cells by repeated pipetting. Next, dissociated cells were pipetted into polylysine-coated culture dishes.

\section{Culture dishes}

Polylysine-coated culture dishes were prepared as follows: each 35 -mm-diameter dish was incubated with 2 to $3 \mathrm{ml}$ of $1 \mathrm{mg}$ of poly-L-lysine/ml (type VI Sigma) in $0.15 \mathrm{M}$ 'Tris (tris(hydroxymethyl)aminomethane) buffer (pH 8.4) for $16 \mathrm{hr}$; then the dishes were washed twice in sterile distilled water followed by a 20 -min rinse in NS and two more washes with distilled water. These were stored dry for up to 2 weeks at room temperature before use. In some experiments, polylysine-coated glass coverslips were used to allow higher resolution microscopic examination. These were prepared by boring a $20-\mathrm{mm}$ diameter hole in the bottom of the culture dish and attaching an acid-cleaned, carbon-coated coverslip with a silicone rubber sealant (Dow Corning). Polylysine then was applied as described above.

Collagen-coated dishes were prepared by incubating culture dishes with autoclaved $0.1 \%$ gelatin (Knox) dissolved in distilled water. After setting overnight, excess gelatin solution was aspirated off and the dishes were stored under desiccation.

CMHL-15 pretreated dishes were prepared by exposing polylysine-coated culture dishes to 1.5 to $2.0 \mathrm{ml}$ of CMHL-15 at room temperature for $24 \mathrm{hr}$. The dish was held either flat to coat the whole surface of the dish or at a $30^{\circ}$ angle to coat only half of the dish surface (Collins, 1978b, 1980). The CMHL-15 was removed and each dish received $2.0 \mathrm{ml}$ of unconditioned HL- 15 for culturing isolated neurons. In some instances, the dishes were washed twice with distilled water prior to the addition of HL-15. 


\section{Neurite measurement}

Dissociated Helisoma central ganglion cells were cultured in 35-mm-diameter polylysine-coated dishes in 2.0 to $3.0 \mathrm{ml}$ of medium per dish. There were approximately 200 to 400 neurons per dish (i.e., three dissociated ganglionic rings per dish) and a small number of non-neuronal cells per dish. Viable neurons were distinguished from other cells by the following criteria: (1) neurons were initially round, spherical cells with an appearance much like that of neurons within an intact ganglion; (2) neurons were large (range 15 to $100 \mu \mathrm{m}$ ) and phase bright; (3) only neurons adhering to the culture dish surface were considered viable; (4) most neurons contained yellow pigment characteristic of molluscan neurons in general. Extensive outgrowth was observed only in cells meeting the preceding criteria. Non-neuronal cells were either much smaller rounded cells or flattened fibroblast or glia-like cells.

Axon initiation and elongation were observed by phase contrast microscopy of living cells. To determine the percentage of sprouted neurons, the entire field of the plastic dish bottom or glass coverslip was examined. A typical dissociation produced 50 to 200 neurons that stuck to the surface of the plate. Any neuron which possessed one or more neurites with a length at least equal to the diameter of the soma was scored as a sprouted cell.

The rate of growth of the axon and the movement of growth cones was determined on neurons cultured on polylysine-coated coverslip tissue culture dishes by photographing individual growing cells or growth cones at various time intervals. Photomicrographs were taken with a Nikon EFM camera mounted on an inverted Wild M40 or fixed stage Leitz Diavert microscope. Analyses of axon growth were performed by using the method of Bray (1973).

Neuritic growth in intact buccal ganglia was observed by whole mount examination of Lucifer Yellow-filled cells. Lucifer Yellow (3\% in distilled water) was injected by steady, hyperpolarizing currents which were less than $10 \mathrm{nA}$ in amplitude and 1 to $2 \mathrm{~min}$ in duration. Specimens were fixed overnight in phosphate-buffered $4 \%$ formaldehyde ( $\mathrm{pH} 7.4$ ) at $4^{\circ} \mathrm{C}$, dehydrated, and cleared in methyl salicylate before examination (Murphy and Kater, 1980a).

\section{Electrophysiological measurements}

Conventional recording and display techniques were used for electrophysiological measurements. Recordings were made via glass filament microcapillaries filled with $4 \mathrm{M}$ potassium acetate which were connected to a unity gain high impedance amplifier and were displayed on a Tektronix 564 storage oscilloscope. The reference electrode was a virtual ground connected to a pulsing circuit for inserting calibration pulses. Isolated neurons were viewed and impaled under phase optics on a fixed stage Leitz Diavert microscope. Micromanipulation was provided by a Licht ultramanipulator (E. Licht and Co.) with bent electrodes. Photographs were taken from the face of the oscilloscope using a Polaroid back Tektronix oscilloscope camera.

\section{Results}

\section{Neuronal growth in vitro}

Organ culture of buccal ganglia and their associated nerve trunks provided one system for assessing the growth of identified neurons during axonal regeneration in vitro. Our standard paradigm was to crush the esophageal nerve trunk approximately $100 \mu \mathrm{m}$ from the buccal ganglia (severing axons within the trunk) and later to assess the regenerative outgrowth of specific identified neurons by injecting them with the fluorescent dye, Lucifer Yellow (Stewart, 1978). Neurons 4 and 5 extend axons down this nerve trunk (Murphy and Kater, 1978; $1980 \mathrm{a}, \mathrm{b})$ and were convenient neurons in which to monitor growth. When ganglia were cultured in vivo following nerve crush (Murphy and Kater, 1980a), significant sprouting and outgrowth were observed along the entire length of esophageal nerve trunk within 2 days of treatment. In vitro, in defined HL-15, significantly less sprouting was observed (Fig. $1, A 1$ and $A 2$ ). The addition of cocultured Helisoma central ganglia (three brains per $\mathrm{ml}$ ) to $\mathrm{HL}-15$ (CCHL-15) allowed more prolific neurite outgrowth (Fig. 1, B1 and B2) along the entire length of the nerve trunk, comparable to in vivo outgrowth. After short culture periods, growth can be quite variable and CCHL-15 cultures are similar to cultures in control HL15 (Fig. 1, $A 1$ and $B 1,1$ day of culture), but, with longer culture periods (Fig. 1: $A 2=6$ days; $B 2=5$ days), differences in the amount of growth in CCHL-15 and HL-15 were readily apparent. In every series, co-cultured preparations were always judged to show more growth than HL-15 controls (eight series, 40 ganglia). This observation also was apparent with other identified neurons in the buccal ganglia. The massive proliferation and great number of sprouts arising from a neuron during regeneration made quantification of these observations impractical in situ and no quantitative statements could be made. A more direct and quantitative assessment of the effect of co-culturing was obtained by isolating and culturing the somata of neurons from the Helisoma central nervous system.

Enzymatically and mechanically isolated neuronal somata maintained their spherical shape when placed into cell culture on polylysine-coated glass or plastic surfaces. There was no apparent difference in the degree of cell flattening with different culture conditions (see below). In defined HL-15, there was rarely any sign of neurite outgrowth (Fig. 2A) although, electrophysiologically, these cells were all viable as described below. In those rare cases where any neuritic outgrowth was observed, it was restricted to a single short neurite. In order to test the effect of CCHL-15, as observed in our organ culture experiments, we cultured isolated neurons with the addition of two intact Helisoma central ganglionic rings per $\mathrm{ml}$ to each culture dish. Under these conditions, dramatically different neuronal morphology was observed (Fig. $2 B$ ); many of the isolated neuronal somata displayed extensive neurite sprouting and non-neuronal cells were observed throughout the cultures (Fig. $2 B$, inset). These non-neuronal cells were presumed to be of fibroblastic or glial origin and their rapid proliferation frequently ob- 

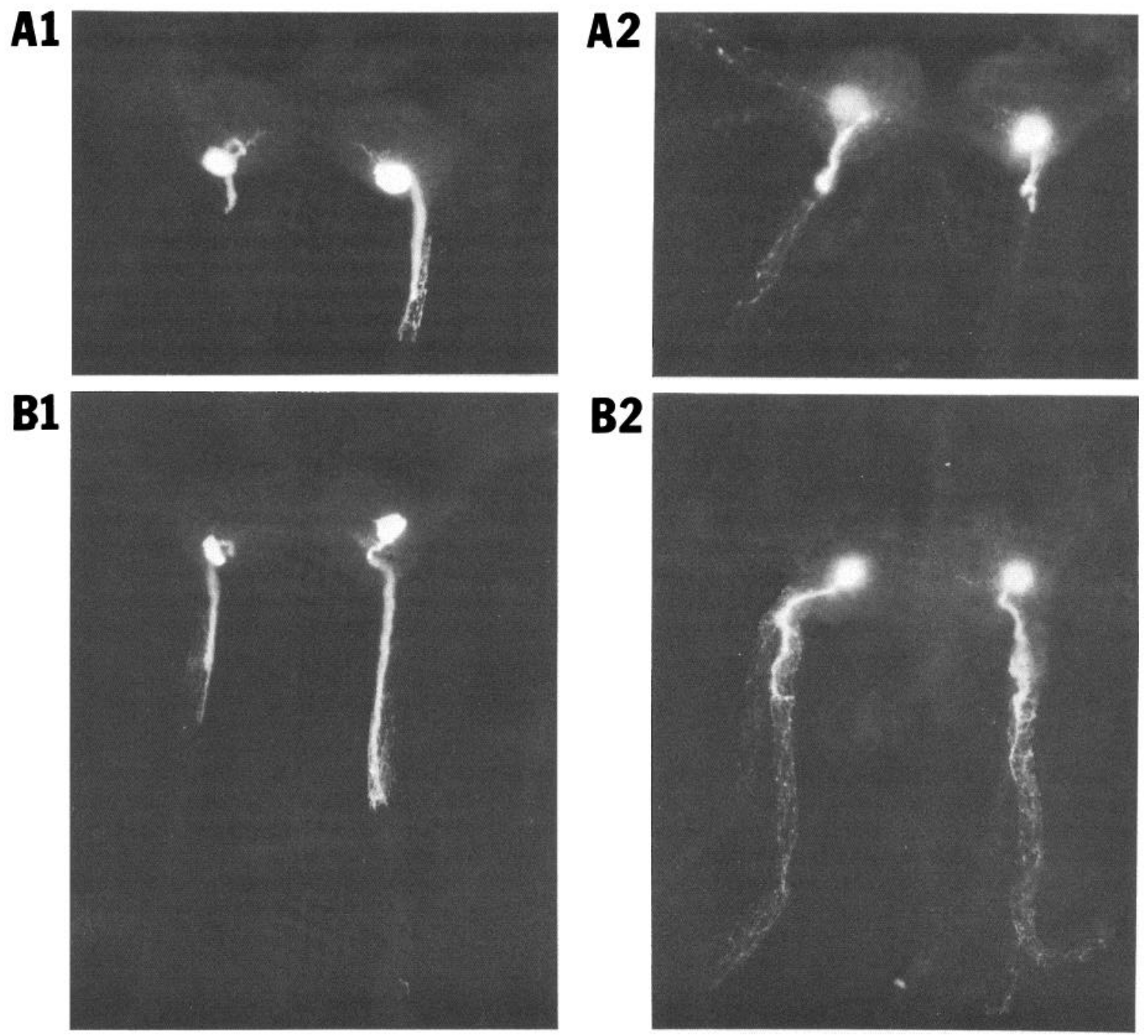

Figure 1. Growth of neurites within cultured buccal ganglia. Neuronal growth is observed within nerve trunks of buccal ganglia after crushing the esophageal nerve trunks. These nerve trunks contain the axons of neurons $5 \mathrm{R}$ and $5 \mathrm{~L}$, shown filled with Lucifer Yellow in all panels of this figure. $A$, Ganglia cultured in control HL-15. The growth in situ after 1 day ( $A 1)$ and 6 days $(A 2)$ in HL-15 can be compared to parallel cultures with co-cultured Helisoma brains $(B)$. B, Ganglia cultured for 1 day $(B 1)$ and 5 days (B2) in HL-15 with co-cultured brains (CCHL-15). Co-culture wells contained one buccal ganglion and one whole central ganglionic mass in $0.33 \mathrm{ml}$ of HL-15. Note the greater amount of growth in CCHL-15, especially at day $5(B>A)$. Every CCHL15 series was judged by a naive observer to exhibit more neuritic outgrowth than HL-15 control series (eight series, 40 ganglia). Neuronal cell bodies are 500 to $600 \mu \mathrm{m}$ apart.

scured neurons and their processes (e.g., neuron in Fig. $2 B$, lower right). These non-neuronal cells were not released during cell dissociation procedures, as cultures of isolated neuronal somata without co-cultures did not contain these cells. On the other hand, simply placing whole central ganglia in culture medium could give rise to a similar non-neuronal cell type covering the bottom of the dishes within 2 to 3 days. These cells need not originate from intact ganglia, since culture of isolated nerve trunks 300 to $400 \mu \mathrm{m}$ in length also resulted in their release and proliferation.

Co-cultured brains could stimulate neuronal outgrowth by releasing a soluble factor into the medium. We produced conditioned medium (CMHL-15) by incubating HL-15 with two Helisoma brains per ml for $72 \mathrm{hr}$ in sterile glass Petri dishes. When CMHL-15 then was employed as the culture medium, isolated neuronal somata produced neuritic outgrowth indistinguishable from that obtained with CCHL-15 (Fig. $3 A$ ). There are two avenues by which conditioning could occur under these conditions: (1) a soluble factor could be released from the ganglia or (2) cells could be released from the ganglia and carried over into our conditioned medium. It is unlikely that cells were carried over, as non-neuronal cells in CMHL-15 cultures were rare. Additionally, simply maintaining the prepared conditioned medium under standard culture conditions without adding dissociated nerve cells did not produce any non-neuronal cells. Filtration of CMHL-15 through Gelman 0.2- $\mu$ m filters did not abolish the factor's activity (Fig. $3 B$ ). This suggested that a 
A

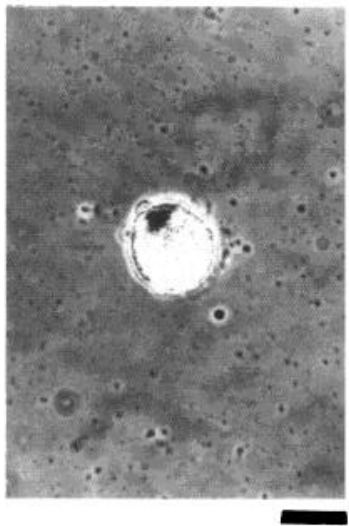

B

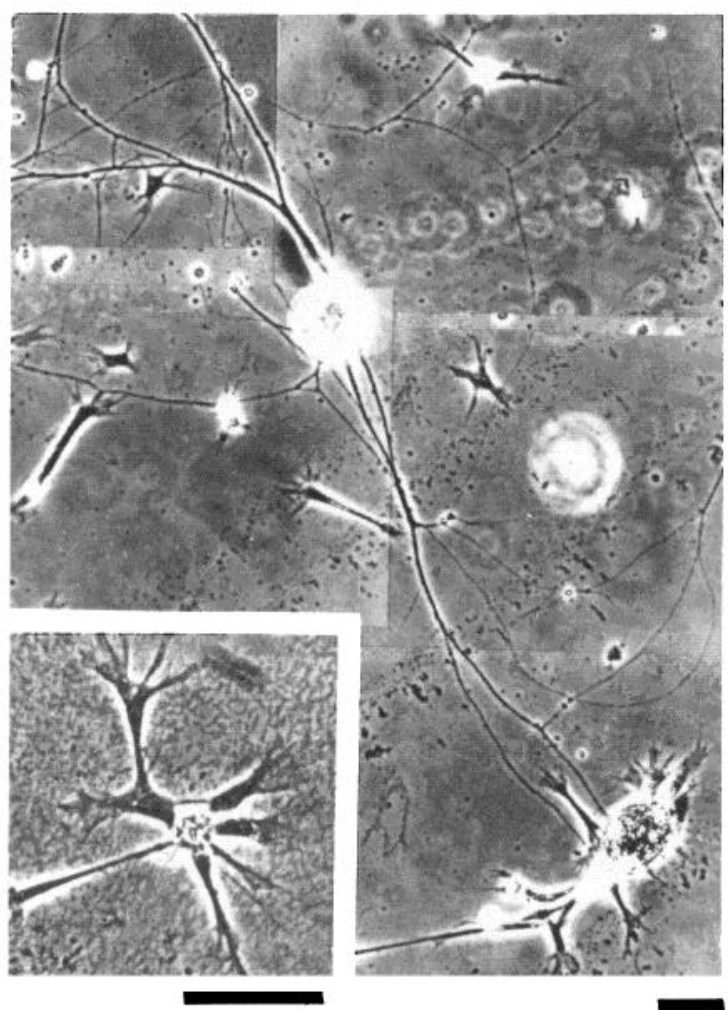

Figure 2. The effects of co-cultured brains upon isolated neurons in vitro. A, A spherical neuron in control HL-15 for 6 days. Limited outgrowth was seen in less than $2 \%$ of neurons grown in HL-15. $B$, A neuron grown in CCHL-15 (two brains per ml of HL-15) for 6 days. Note the extensive outgrowth from this single neuron. In addition to neurons, the culture contained a large number of non-neuronal cells of fibroblastic or glial origin (inset). These cells eventually overran the culture dish, often obscuring neurons and their processes ( $B$, lower right). Scale bars $=50 \mu \mathrm{m}$.

factor(s) other than cells promoted neurite outgrowth in CMHL-15. An interesting feature of this conditioning factor (CF) is its apparent affinity for the cellulose nitrate used in Millipore filters but not for the cellulose triacetate used in Gelman filters of comparable sizes. Filtration of CMHL-15 through $0.22-\mu \mathrm{m}$ Millipore filters blocked all subsequent neuronal sprouting (Fig. $3 C$ ). Fresh HL-15 incubated with CF-adsorbed Millipore filters for $24 \mathrm{hr}$ did not show any sprouting activity, thus suggesting that CF was bound tightly rather than simply filtered out. Identical results were obtained with unwashed filters and ones prewashed in hot saline. Taken together, these observations are consistent with the idea that $\mathrm{CF}$ is a noncellular, soluble substance(s) that binds to specific substrates. Further evidence for CF adsorption is presented later.

The number of neurite-bearing neurons in CMHL-15 increased with time (Fig. 4, $\square$ ). By day $2,13 \%$ of neurons had significant neuritic outgrowth, and by day 4 , this number had reached $17 \%$. Longer term cultures, although not assayed quantitatively, indicated that progressively more neurons sprout with time and that neuritic outgrowth was asynchronous under these conditions. A similar but apparently delayed time course was observed for neurite outgrowth in CCHL-15 (Fig. 4, ๑). There appears to be a lag of about 1 day such that the co-culture values on day 1 were not significantly different from controls $(\boldsymbol{\Delta})$. This may indicate that the co-cultured brains required time ( $\simeq 1$ day) to release CF. CMHL-15 neurons produced significantly greater neuritic outgrowth than neurons grown in fresh HL-15 on all days $(\boldsymbol{\Delta})$. The similarity of the shapes of the CCHL-15 and CMHL-15 curves may indicate that, in both cases, the neurons were responding to the same cue(s).

\section{Growth properties}

While the characterization of the nature of in vitro neuritic outgrowth has been well documented for vertebrate neurons, the present communication represents one of the few demonstrations of in vitro neuritic outgrowth in invertebrates. We have compared essential features of neuritic growth properties in Helisoma to those of vertebrate neurons. The rapidity of growth and complexity of a typical pair of intertwining Helisoma neurons in CMHL-15 is illustrated in Figure 5. In the 20.5 $\mathrm{hr}$ intervening between photograph $A$ and photograph $C$, the two neurons had undergone significant changes, with the addition of secondary, tertiary, and quaternary branches. Because of the complexity of these neurons' morphologies, it was difficult to derive a single measure of growth rate for the processes. The rates for some individual neurites could be calculated, however; for ex- 

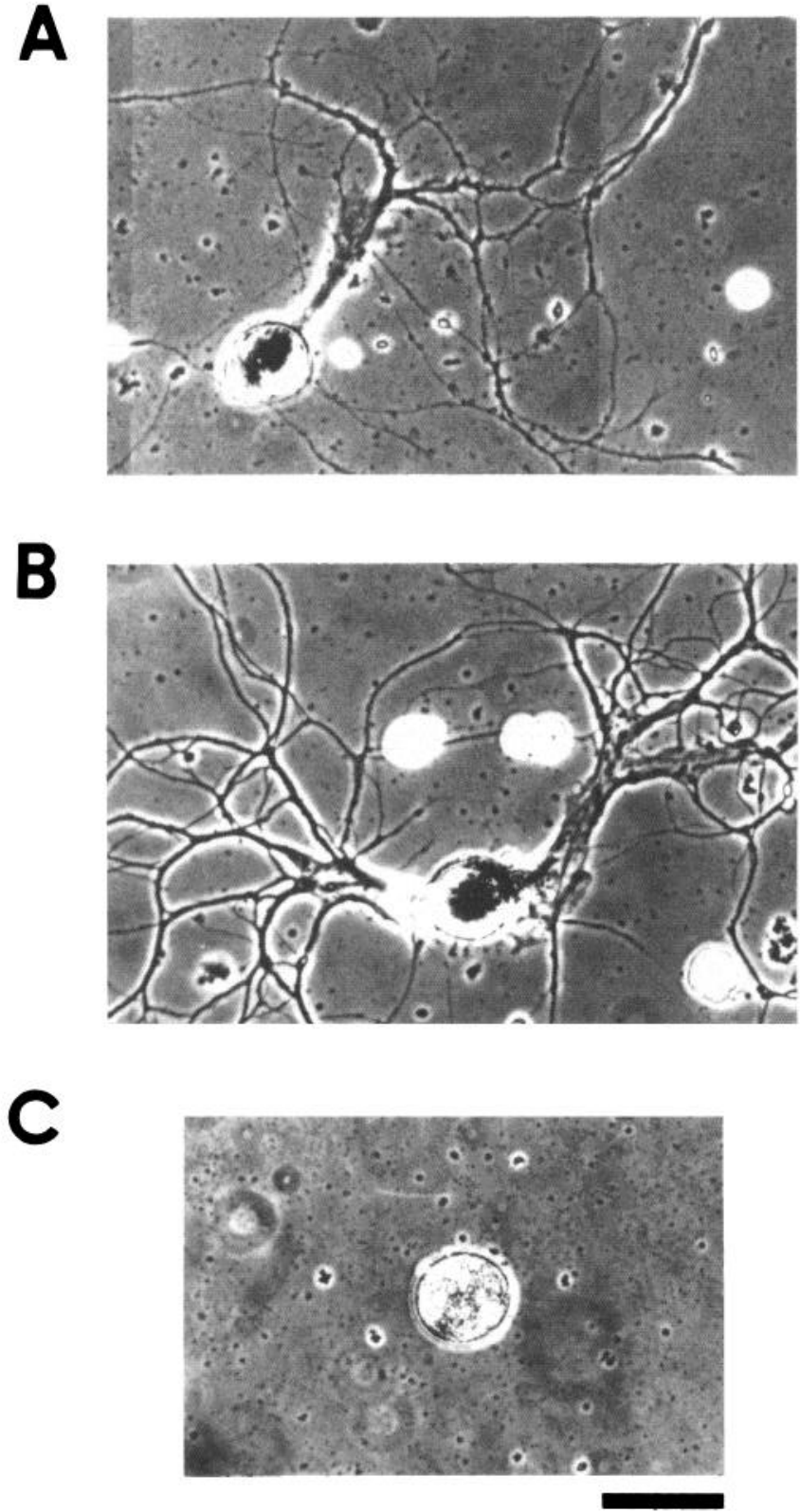

Figure 3. The effect of conditioned medium on isolated neurons. Medium conditioned by the incubation of two brains per $\mathrm{ml}$ in HL-15 (CMHL-15) in sterile glass dishes for $72 \mathrm{hr}$ promotes the outgrowth of neurites. $A$, A neuron cultured for 3 days in CMHL-15. Quantitative experiments show that $16.6 \%$ of neurons in CMHL-15 had neurites at 4 days (Fig. 4). B, A neuron cultured 4 days in CMHL-15 filtered through a Gelman $0.2-\mu \mathrm{m}$ filter. No significant decrease in activity of the medium was observed (9.2\% sprouting at 4 days). $C$, A neuron cultured 6 days in CMHL-15 filtered through a Millipore $0.22-\mu \mathrm{m}$ filter. Neurite production was reduced to control HL-15 values at 4 days $(0.65 \%$; see also Fig. 4). Scale bar $=100 \mu \mathrm{m}$.

ample, the neurite at 12 o'clock (Fig. $5 A$ ) had a minimum rate of growth of $19 \mu \mathrm{m} / \mathrm{hr}$.

Time lapse still photographs allowed a comparison between neuronal growth in Helisoma and the fundamental data on mammalian neuronal outgrowth provided by Bray (1973). Bray's basic conclusion was that, once formed, an axon adds new length only at its growing tip (i.e., the growth cone). Our measurements yielded the same conclusion for two cultured Helisoma neurons. Initial observations were begun after primary axonal outgrowth had been initiated (not as in the case of Bray who started with spherical neurons and used time lapse cinematography). In one neuron (Fig. 6), over a period of nine observations spanning $19.5 \mathrm{hr}$, free endings, 1,2 , and 3 , continued to grow while the nodal distances, $A, B, C$, and $D$, remained constant. The short neurites located at branch points $C$ and $D$ failed to elongate after initiation. These growth characteristics were precisely those observed by Bray for mammalian neuronal outgrowth and were repeated in a second analysis of a second preparation (not shown). Further analysis of growth rates of the neuron in Figure 6 indicated an eventual decrease in growth rates. All three neurites exhibited a uniform rate of growth up to $675 \mathrm{~min}(26.6 \mu \mathrm{m} / \mathrm{hr})$. After $675 \mathrm{~min}$, the rates diminished to 11.9 (neurite 1), 17.1 (neurite 2), and $5.6 \mu \mathrm{m} / \mathrm{hr}$ (neurite 3). Such decreased growth rates led to the eventual stabilization of morphology for most neurons.

The preceding results demonstrate that growth cones in a mollusc are a means of axonal elongation as in vertebrate neurons. An indication of the dynamic movements of these molluscan growth cones is provided by the time sequence of 35 min shown in Figure 7. Over this duration, the growth cone had advanced $30 \mu \mathrm{m}$ and had given rise to what became a major branch point along a neurite. Additionally, rapid activity of the veils and microspikes at the tip of the growth cone and along the shaft of the axon was prominent. Movements of individ-

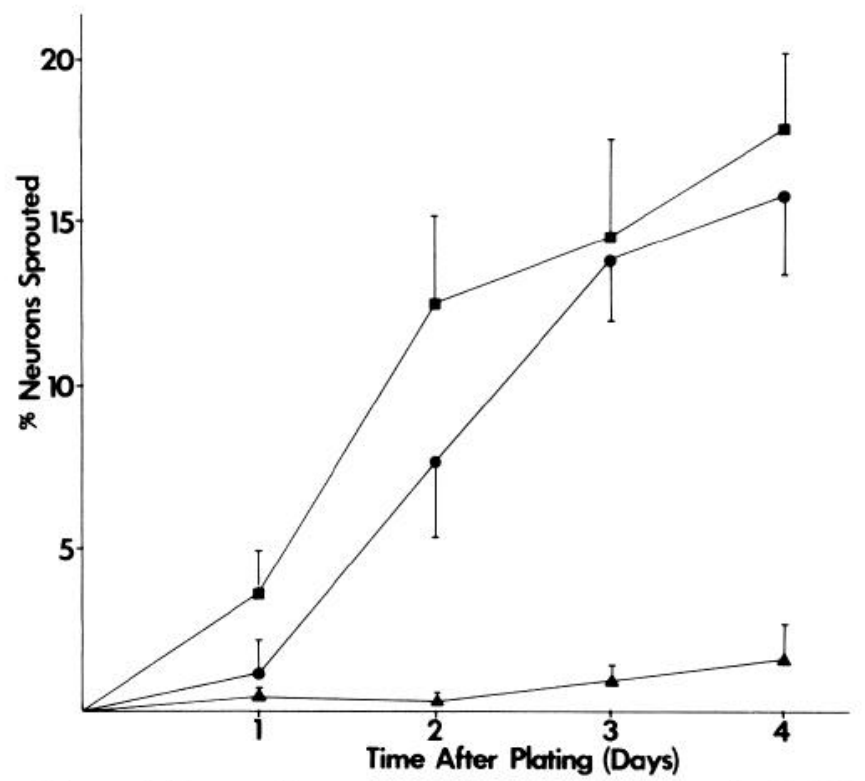

Figure 4. Comparison of growth media. Cultures in CMHL15 (ם) were scored as the percentage of adhering neurons which had sprouted neurites of a length greater than the soma diameter and compared to control cultures in HL-15 ( $\boldsymbol{\Delta})$. Differences from control (HL-15) were significant on all days for CMHL-15 (day $1, p<0.02$; day $3, p<0.01$; days 2 and $4, p<0.001 ; t$ test). The results of experiments with CCHL-15 (O) are included. Differences from HL-15 were significant at days 2,3 , and 4 (days 2 and $4, p<0.01$; day $3, p<0.001 ; t$ test). All points are plotted as mean \pm SEM; $n=4$ to 7 . 

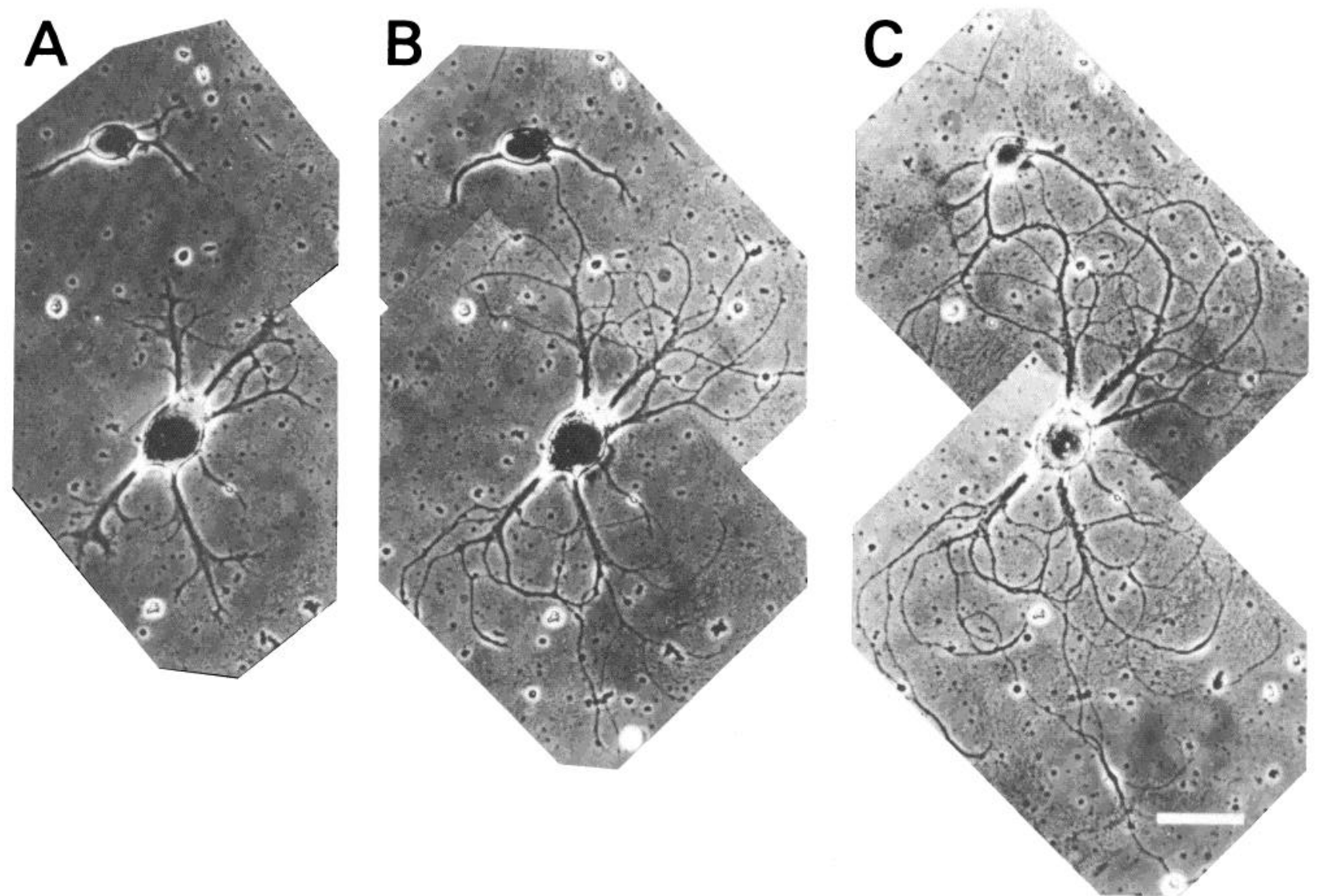

Figure 5. Rate of neurite outgrowth in neurons cultured in CMHL-15. The neurons were plated initially as spherical cell bodies with no carryover of axon segments. $A, 20 \mathrm{hr}$ after the initial plating of neurons on a polylysine-coated dish in CMHL-15. Neurites have extended from a pair of neurons and show characteristic large growth cones and multiple branching. $B$, Same neurons $5.5 \mathrm{hr}$ after $A$. The calculated rate of advance for the first neurite to establish contact on the upper neuron was $19 \mu \mathrm{m} / \mathrm{hr}$. Note that many other neurites also were growing but not all at the same rate. Outgrowth in the upper neuron was much less than in the lower. $C, 20.5 \mathrm{hr}$ after $A$. There was continued elaboration of a complex network of neurites, resulting in intertwining between the two neurons. Scale bar $=80 \mu \mathrm{m}$.

ual microspikes could be observed in periods of less than 1 min. An important determinant of a growing neurite's pathway (and thus the overall morphology of the growing neuron) is the adhesiveness of the substratum (Letourneau, 1975a, b; Bray, 1973). The differential morphologies of vertebrate neurons cultured on collagen compared to neurons grown on polylysine substrates are well recognized. Collagen substrates give rise to neurons with long straight neurites and branches at equal, large angles off main shafts (Bray, 1979), while more adhesive polylysine substrates produce "wandering" neurites and branches at odd angles. Strikingly similar differential morphologies also were observed for Helisoma neurons in CMHL-15 on these two substrata (Fig. 8). Taken together, the neuritic growth rates and morphologies indicated that growth mechanisms of molluscan neurons in cell culture were the same as those described at this level for vertebrate neurons in culture.

\section{Electrophysiological properties of cultured neurons}

Typically, there is a wide variety of electrophysiological characteristics among identifiable molluscan neurons
(Kater et al., 1975; Kandel, 1976). The present investigation was concerned with the culture of unidentified neurons in which we performed an electrophysiological survey of five general classes of neurons: (1) HL-15 spherical neurons, (2) spherical neurons in CCHL-15, (3) spherical neurons in CMHL-15, (4) neurons with prolific neurite outgrowth in CCHL-15, and finally, (5) neurons with prolific neurite outgrowth in CMHL-15.

It is clear that a brain-derived factor(s) was not required for general maintenance of electrical excitability of isolated neurons. Figure $9 A$ shows a spherical neuron cultured 4 days in HL-15 which supported normal, overshooting action potentials. These action potentials exhibited substantial broadening when fired at $0.33 \mathrm{~Hz}$ (Fig. $9 A$, lower trace). Since none of the cultured neurons were of a known type, it was not clear whether this characteristic was a maintained property possessed by the neuron previous to isolation or whether it was developed in response to isolation of the neuronal soma in vitro. Similar results were found for spherical neurons in CCHL-15 (not shown) and spherical neurons in CMHL15 (Fig. 9B). In our survey, a wide range of action 


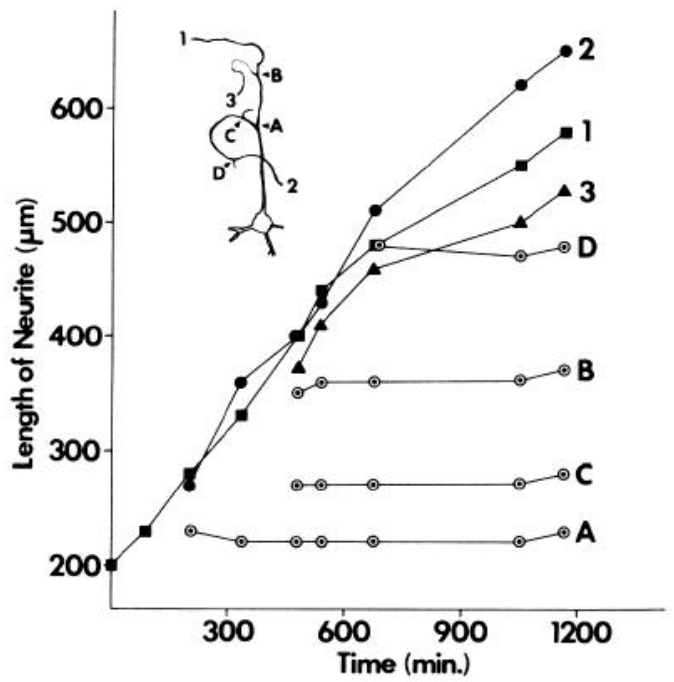

Figure 6. Analysis of neurite extension. Following the analyses of Bray (1973), distances between the soma and either branch points $(A, B, C$, and $D)$ or free ends $(1,2$, and 3) (inset tracing at $1170 \mathrm{~min}$ ) were measured in time lapse photographs. The analysis shows that elongation occurs only at free ends of neurites. Soma to branch point distances $A, B, C$, and $D$ remained nearly constant, while soma to free end distances increased with time (curves 1,2, and 3). Note that the initial growth rates of different branches are nearly identical. From time 0 to $675 \mathrm{~min}$, the regression fit growth rate is $26.6 \mu \mathrm{m} / \mathrm{hr}$. After $675 \mathrm{~min}$, rates lessen to 11.9 (neurite 1), 17.1 (neurite 2), and $5.6 \mu \mathrm{m} / \mathrm{hr}$ (neurite 3 ).

potential characteristics was observed (e.g., not all spherical neurons showed spike broadening). In a limited number of cases of multiple microelectrode penetration over 2 - to 3-day periods, relocatable individual neurons showed the same electrophysiological characteristics; i.e., the neurons had electrophysiologically stable identities. All impaled neurons in every culture condition were capable of producing action potentials and could fire repetitively in response to long depolarizing currents (Fig. 10, $A$ and $B$ ). Neurons with extensive neurites often exhibited depolarizing inflections in response to subthreshold current injections (Fig. 10, $A$ and $B$, left traces). These inflections may have represented local responses in the soma or may have been at a distance from the soma within the neuritic field. We have no direct information on the excitability of the neurites themselves. In general, for all neurons, input impedances were high, in most cases of the order of 100 megohms. A few neurons displayed graded action potentials, but this frequently correlated with low resting potentials, indicating a poor penetration. There was no correlation of this property with any of the specific conditions under which cells were cultured. The only property which might be correlated with a particular condition is that of spike broadening on repetitive stimulation. This characteristic was not seen in neurons with extensive processes. This observation, however, may have simply represented a sampling error. In the context of our overall findings, it was clear that maintenance of basic electrophysiological characteristics of neurons was not dependent upon any exogenous fac- tor(s), while neuritic outgrowth from the same neurons in vitro was dependent upon brain-derived conditioning factor(s).

\section{Adsorption of conditioning factor to polylysine}

A growing body of literature from vertebrate neuron cell culture studies indicates that at least some neurons initiate outgrowth in response to a conditioning factor associated with the substratum. Such surface-associated factors have been demonstrated for dissociated embryonic parasympathetic neurons in chick (Collins, 1978b, 1980; Adler and Varon, 1980). Our initial tests for a surface-associated CF involved preincubating plastic polylysine-coated dishes with CMHL-15 for $24 \mathrm{hr}$. The CMHL-15 supernatant then was removed completely and the incubated dish was covered with fresh HL-15. The CMHL-15 supernatant was, in turn, plated on a fresh polylysine-coated dish. To both sets of plates (CMHL-15 coated dish and CMHL-15 supernatant dish)
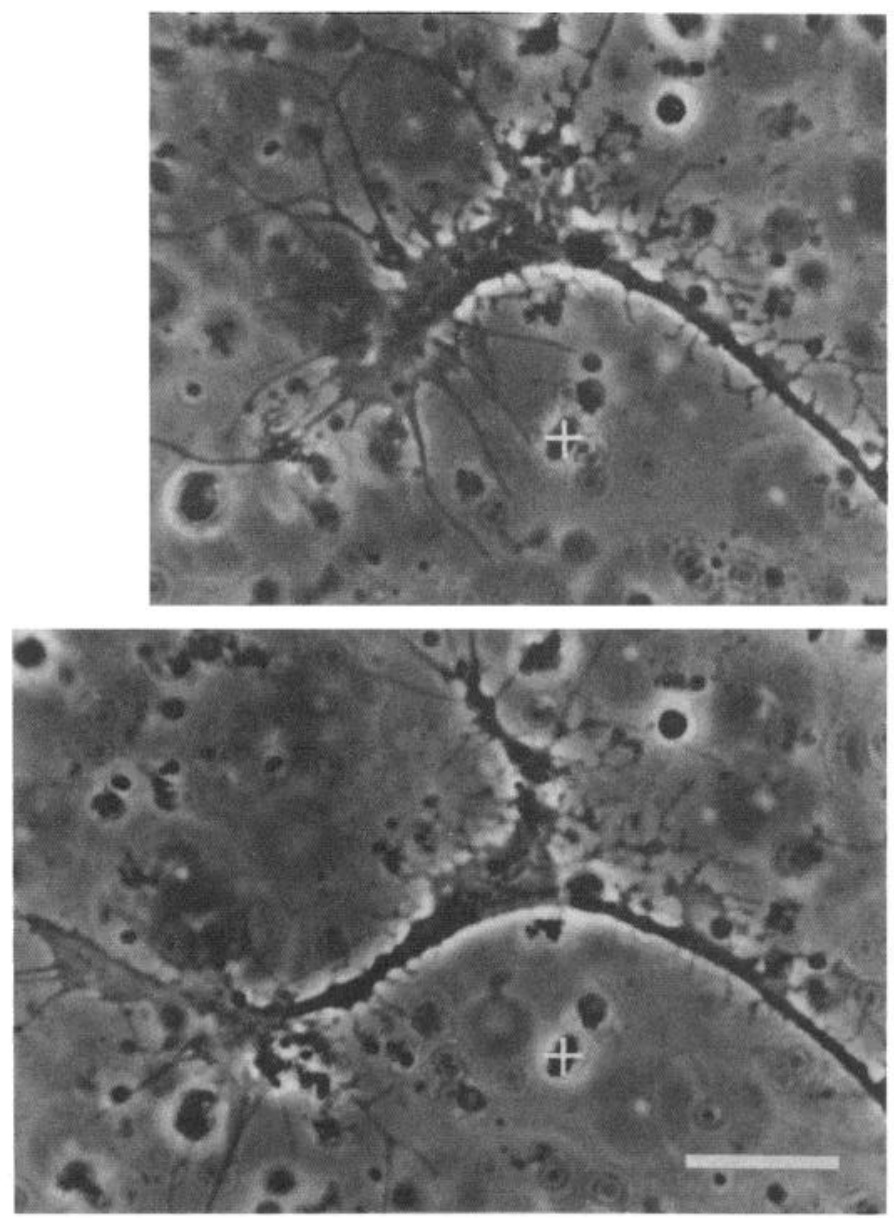

Figure 7. Growth cone movement. Upper panel, A growth cone of a neuron in CMHL-15 $45 \mathrm{hr}$ after initial plating. Lower panel, The same growth cone 35 min later. It has advanced approximately $30 \mu \mathrm{m}$ and a branch point is incipient at the upper part of the panel. Movement can be judged by comparing the growth cone position with stationary particles on the surface of the dish (+). Points of axon attachment appear as flattened membrane along the shaft of the axon. Scale bar $=20 \mu \mathrm{m}$. 
A

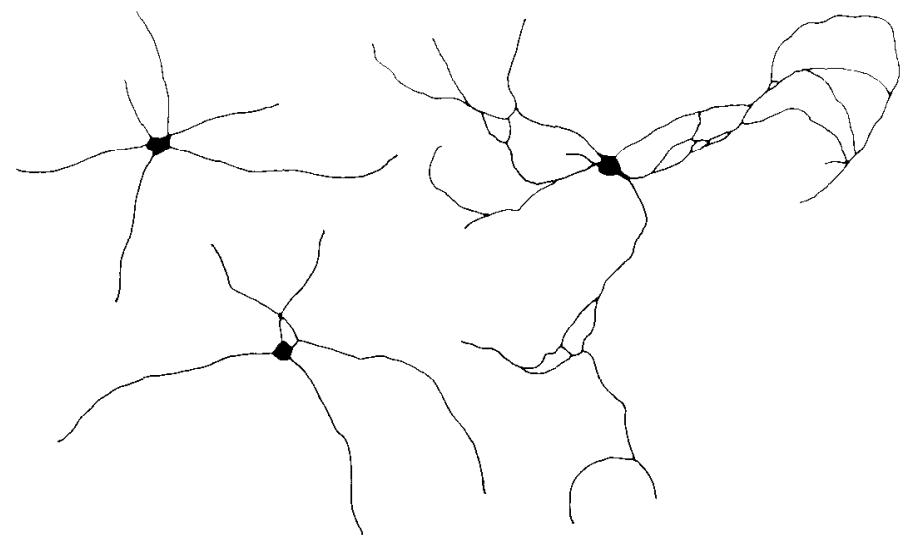

B

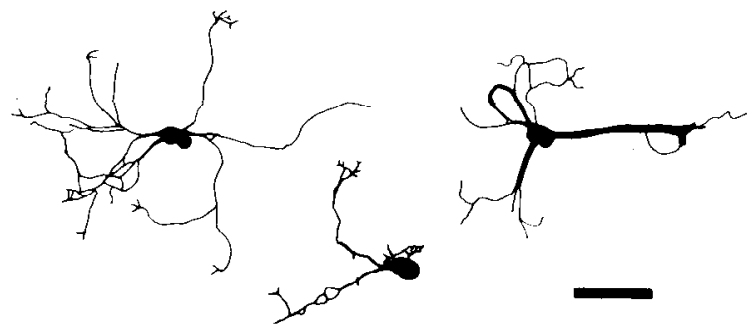

Figure 8. Neuronal morphology on different substrates. The tracings were taken directly from photomicrographs. $A$, Collagen substrate produces neurons with thin, relatively straight neurites attached only at the growth cones. Branching is infrequent and at large angles. The tracing on the right illustrates an exceptional neuron on collagen which branched much more frequently than most other neurons. $B$, Polylysine substrate yields thicker neurites which branch profusely and follow "wandering" paths. These neurites are anchored firmly to the substrate over their entire length. Scale bar $=100 \mu \mathrm{m}$.

and to control plates (HL-15), freshly dissociated cells were added. Under these conditions, neurite outgrowth on the pre-coated plates was significantly different from control ILL-15 (Fig. 11, $n=3$; day $1, p<0.001$; day $2, p$ $<0.002$; day $3, p<0.002$; and day $4, p<0.03$ ). Sprouting also was observed in the supernatant CMHL-15. When the plates containing the supernatant CMHL-15 were scored and summed with those of the CF-adsorbed dishes, the additive curve was found to be nearly superimposable with that of the CMHL-15 curve. Thus, CF activity in CMHL-15 was partitioned between the polylysine surface and the supernatant after 1 day of incubation.

In another series of experiments, polylysine-coated plastic culture dishes were prepared in which only half of the surface was exposed to CMHL-15 for $24 \mathrm{hr}$. The CMHL-15 then was removed completely and freshly dissociated cells were added to the entire plate in fresh HL-15. In each experiment, the neurite outgrowth after 5 days in culture was significantly greater on the CFadsorbed side than on the control side (Fig. 12: experiment I, $p<0.002$; experiment II, $p<0.01$ ). These results demonstrated that $\mathrm{CF}$ could act in association with the surface of the culture dish and that $\mathrm{CF}$ was bound tightly to polylysine rather than acting in soluble form in the conditioned medium. The implication of CF surface association as a mechanism of conditioned media's effects on neuritic outgrowth draws an additional parallel between molluscan neurons and their phylogenetically distant vertebrate counterparts.

\section{Discussion}

Past studies in our laboratory using identified neurons of Helisoma have addressed the questions of normal neuronal circuitry (Kater and Rowell, 1973; Kater, 1974), specific neuronal sprouting and regeneration (Murphy and Kater, 1978, 1980a), neuronal pathfinding (Murphy and Kater, 1980b), changes in central neuronal connectivity (Bulloch et al., 1980), and mechanisms of selection of specific neuronal connections (Bulloch and Kater, 1981). These studies all used the technique of in vivo organ culture (implanting organs of interest into the hemocoel of host snails). In order to study these plastic changes more easily, we have devised an in vitro system for culture of Helisoma neuronal explants (Hadley and Kater, 1979; Kater and Hadley, 1980; R. D. Hadley, R. G. Wong, S. B. Kater, and D. L. Barker, manuscript in preparation). The work reported here was aimed at establishing a quantitative study of neuronal growth as an initial approach to understanding the mechanisms of neuronal connectivity at the cellular level. Our primary observation is that, in both ganglionic organ culture and isolated neuron culture, there exists a $C F$ which promotes neurite outgrowth. We have found further that: (1) growth characteristics of adult molluscan neurons in vitro conform to established concepts of vertebrate neuronal growth in vitro; (2) CF is required for outgrowth of isolated neurons but (3) has no apparent effect on maintenance of electrical excitability; and finally, (4) Helisoma CF can exert its effects on outgrowth from isolated neurons by associating with the substratum.

\section{Growth characteristics of isolated Helisoma neurons}

Our initial observation of a CF was the effect of cocultured brains upon growth in vitro in organ culture of buccal ganglia. Although neurite outgrowth occurs within ganglia in the absence of CF, that growth is never as great as that observed in vivo. Other authors have made parallel observations using co-cultured tissues in vitro. Chen and Levi-Montalcini (1970) showed that explanted embryonic foregut tissue was necessary for in vitro fibrillar growth from embryonic neurons of the cockroach Periplaneta americana. Pollack and Liebig (1977) showed that differentiating limb bud tissue had a trophic influence on cultured frog spinal cord explants. Conditioning the medium in Helisoma enhanced neurite outgrowth in cultured buccal ganglia to levels comparable to in vivo growth. This growth in vitro is sufficient to allow formation of novel neuronal connections as described by Bulloch and Kater (1981) for in vivo cultured ganglia (R. D. Hadley, R. G. Wong, S. B. Kater, and D. L. Barker, manuscript in preparation).

In vitro organ culture has allowed us a higher degree of manipulative freedom while retaining the normal substrate for neuronal interaction (i.e., the intact ganglion). Because of the complex three-dimensional nature of the 


\section{A}
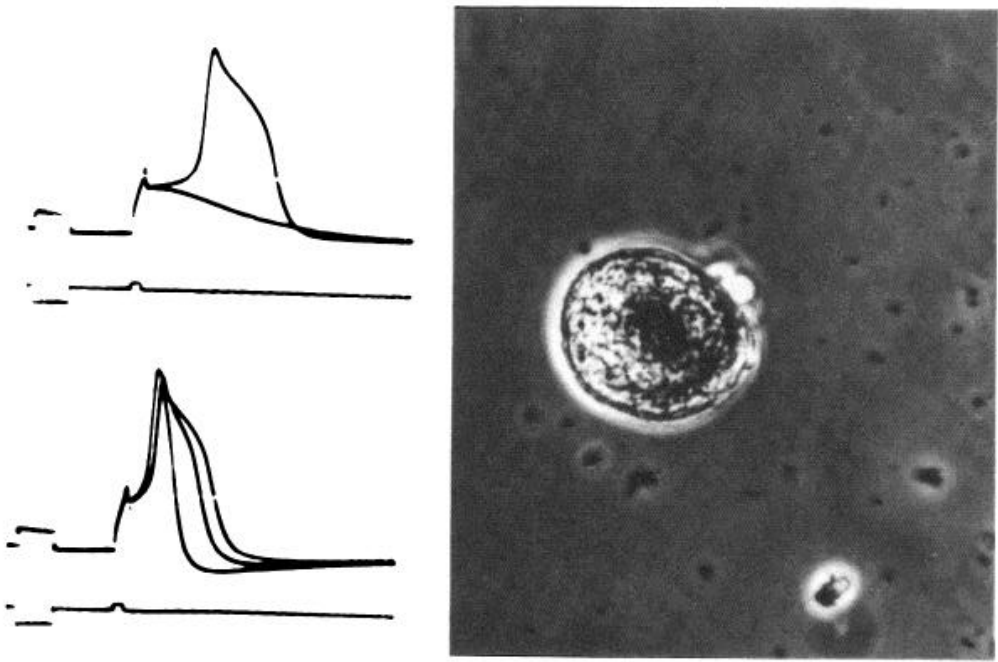

B
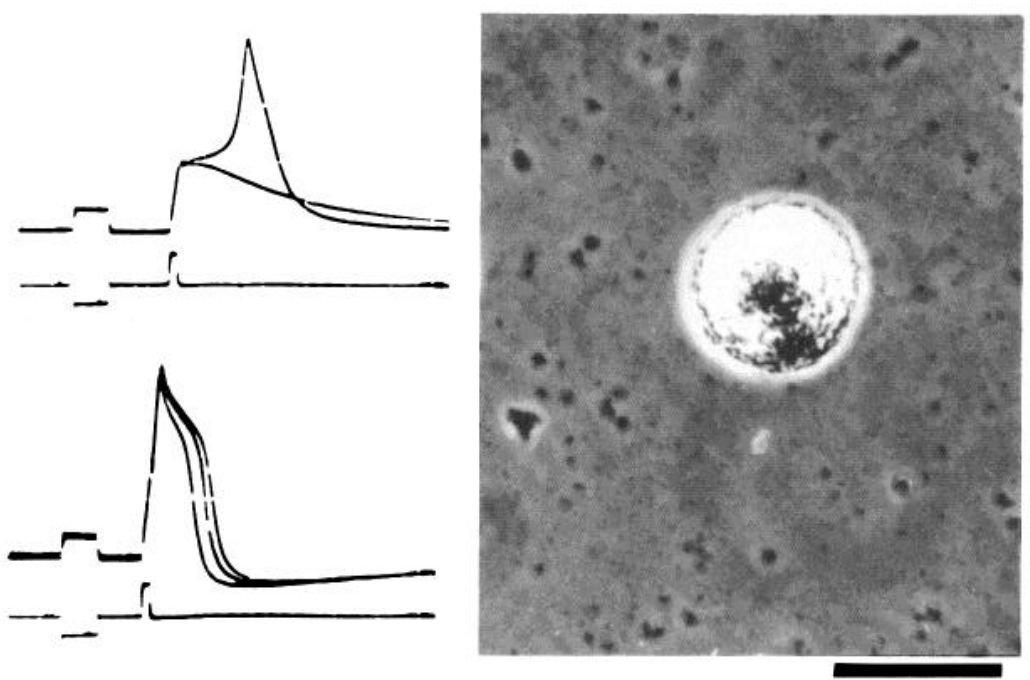

Figure 9. Electrical activity of spherical neurons. $A$, Intracellularly evoked action potentials in a neuron (shown at right) cultured 4 days in CMHL-15. The current injection (monitor, lower traces) gives rise to overshooting action potentials (upper traces). Repetitive stimulation at $0.33 \mathrm{~Hz}$ reveals a facilitated broadening of the action potential (lower traces). $B$, Nearly identical response of a neuron (right) cultured in control HL-15 for 6 days. Square calibration pulses at the beginning of each trace: $10 \mathrm{mV}, 50 \mathrm{msec}, 0.8 \mathrm{nA}$. Scale bar $=50 \mu \mathrm{m}$.

substratum in the ganglion however, quantification of the dynamics of neuronal morphology is unsatisfactory in organ culture. Cell culture, on the other hand, obviates many of these limitations.

Initially, isolated Helisoma neurons were cultured in $\mathrm{CF}$ derived from co-cultured brains. This promoted excellent neurite outgrowth (Fig. 2), however, it introduced rapidly proliferating non-neuronal cells. The nature of these cells is unknown although they show phagocytic behavior toward debris and dead neurons in cultures. Similar properties have been described for the microglia that proliferate after axotomy of mammalian neurons (Jacobson, 1978). The role of these non-neuronal cells in promoting neurite outgrowth is unclear although they are always present during the production of CMHL-15. Banker (1980) reported that astroglial cells in culture release factors into the medium that promote the growth and survival of rat hippocampal neurons in vitro. Although it is not clear that CMHL-15 promotes neuritic outgrowth in the absence of any non-neuronal cells, the question of whether the conditioning factor in Helisoma may be a by-product of these cells has not been determined. However, it is noteworthy that the trophic factor reported by Banker (1980) for rat astroglial cells differs in its mode of action, as it does not bind to the substrate in amounts sufficient to promote nerve growth when conditioned medium is replaced by control medium.

When initially plated, all cells start as spheres with a 
A
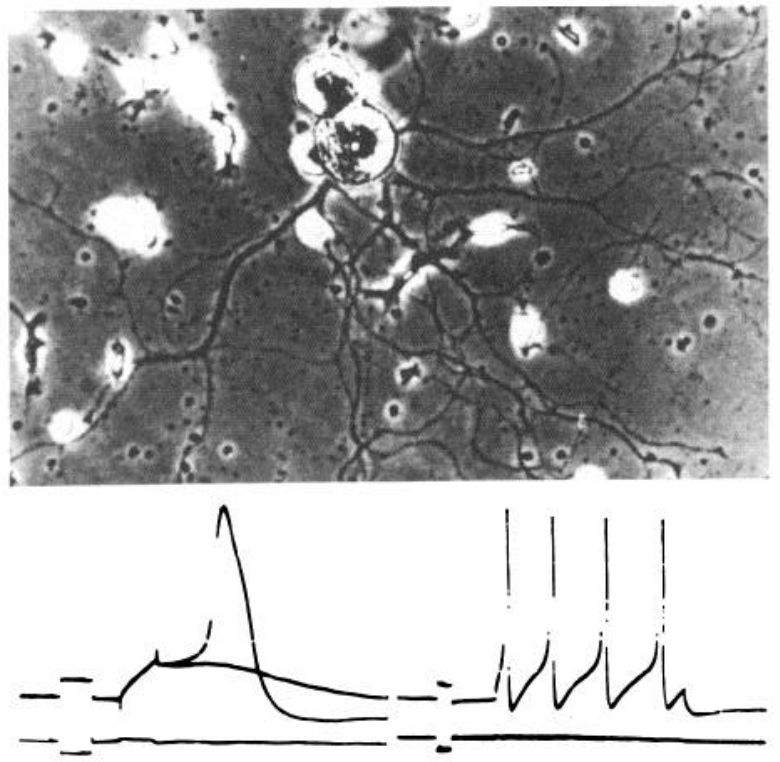

B

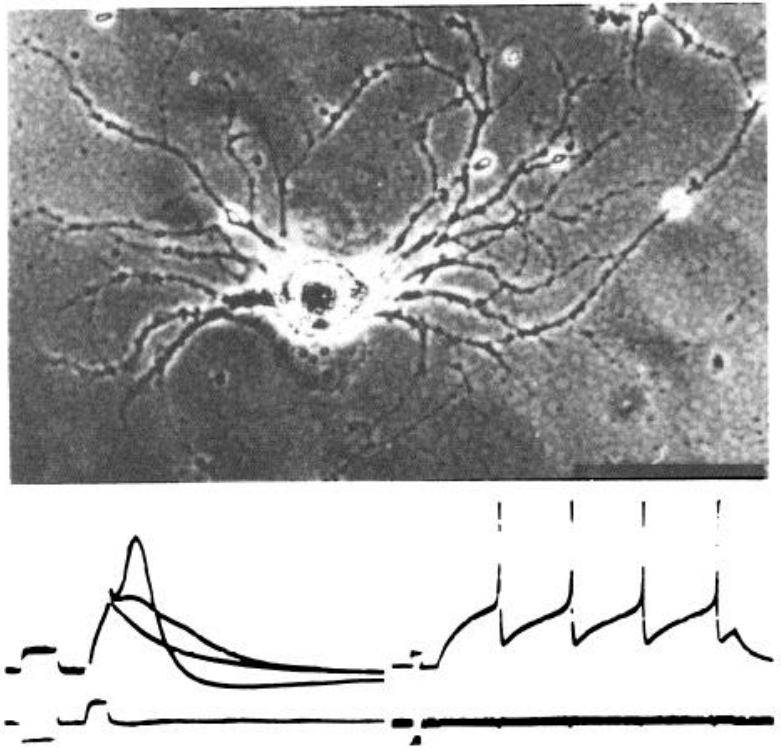

Figure 10. Electrical excitability of sprouted neurons. A, A neuron cultured 6 days in CCHL-15 with extensive neurites (top) produced action potentials in response to intracellular injection of short (left) or long (right) depolarizing currents. Some subthreshold currents gave rise to small local responses (left). Repetitive spiking occurred atop long depolarizing pulses. $B$, Similar responses in a neuron cultured 8 days in CMHL-15 (above). Subthreshold current injections (left) produced waveforms with multiple depolarizing inflections. Square calibration pulses at the beginning of each trace: $10 \mathrm{mV}, 10 \mathrm{msec}$ for left traces; $50 \mathrm{msec}$ for right traces; $0.8 \mathrm{nA}$. Scale bar $=100 \mu \mathrm{m}$.

few having very short axon stumps (less than 1\%). The question of whether CF acts as an initiation or elongation factor has not been determined. However, we have seen the formation of circular veils and very short extensions on neurons in control plates. Collins (1978a) has interpreted similar veils on chick ciliary ganglion neurons as manifestations of neurite initiation. Perhaps Helisoma

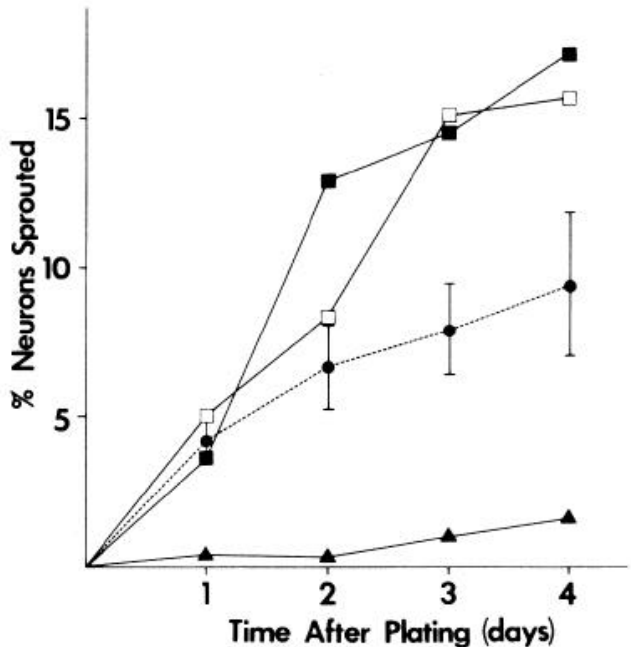

Figure 11. Adsorption of CF to polylysine substrates. Polylysine-coated dishes were incubated for $24 \mathrm{hr}$ with CMHL-15. Then CMHL-15 was removed and reserved as "supernatant CMHL-15." Two groups of isolated neurons were assayed for sprouting. One group was cultured in fresh HL-15 on the preincubated plates and the other group was cultured in supernatant CMHL-15 on fresh plates. CF-adsorbed plates $(9$, mean \pm SEM) promoted significant sprouting compared to controls $(\boldsymbol{\Delta}, p<0.002$ on days 1 to $3 ; p<0.03$ on day $4 ; t$ test). The combined sprouting activity of CF-adsorbed dishes and supernatant CMHL-15 dishes (sum of activities, $\square$ ) was similar to sprouting in CMHL-15 (ם). Control and CMHL-15 data are replotted from Figure 4.

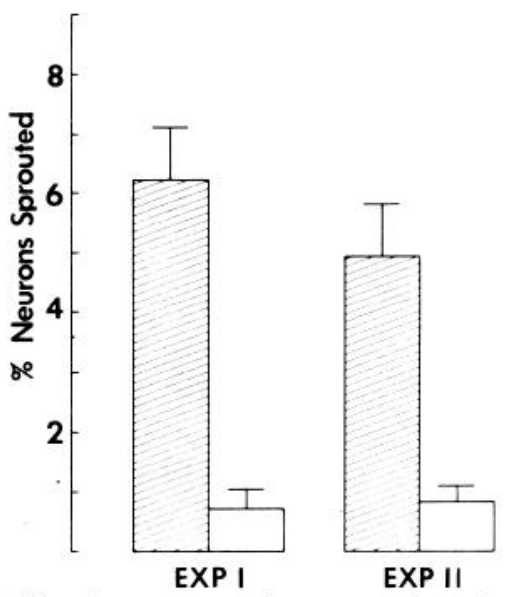

Figure 12. Neurite outgrowth on restricted regions of adsorbed CF. When only one-half of the surface of a culture dish was preincubated with CMHL-15, neurons attached over the entire surface of the plate but showed extensive outgrowth only on the pretreated half. Neurons were scored for outgrowth 5 days after being plated. In each experiment, neurite outgrowth was significantly different on the CF-coated half of the plate (shaded bar) compared to the untreated side (experiment I, $p$ $<0.002$; experiment II, $p<0.01 ; t$ test). Vertical lines on each histogram represent SEM $(n=4)$.

neurons, as chick ciliary ganglion neurons, require $\mathrm{CF}$ for elongation but not for initiation of neurites.

Helisoma neurons appear to reach a stable morphology after 3 to 5 days of outgrowth. Initial outgrowth involves the formation of major neuritic branches which set the 
primary morphological pattern for subsequent growth (e.g., monopolar, bipolar, or multipolar). Through the elaboration of secondary, tertiary, etc. branches, a finite form ultimately is reached. In one exemplary case, a neuron which first sprouted 3 days after plating reached a stable form within 2 days and maintained this form until the culture was terminated at 15 days. The rate of growth for individual neurites diminishes with time. For the neuron described in Figure 6 , the average growth rate of the various neurites is reduced by more than half after $675 \mathrm{~min}$. Such decreased rates occur even when neighboring neurons are in their rapid growth phase. These observations are consistent with previous observations made on isolated buccal ganglia cultured in vivo (J. F. Stamler, S. B. Kater, A. D. Murphy, and A. G. M. Bulloch, manuscript in preparation). These isolated ganglia produce a neuroma consisting of profuse fine neuritic branches. The neuroma increases in net size for up to 3 weeks, after which, no further net increase is observed. These two sets of observations suggest that individual neurons may have a finite capability for net growth. After a certain amount of neuritic outgrowth is attained, only small changes and readjustments of fine processes may occur.

It is apparent that the neurite-bearing cells in CFcontaining cultures are a minority population at 4 days (15 to $20 \%$ ). It is clear from longer term observations (not shown quantitatively) that, although some neurons had reached stable morphologies, no steady state level of neurite expression had been attained at this time; additional neurons were still sprouting. The failure of some neurons to sprout may be due to a relationship between the CF titer and outgrowth as suggested by Figure 11. The values for CF-adsorbed plates are roughly half of those of CMHL-15 plates at day 4 . Increased levels of CF may allow increased levels of outgrowth or even total outgrowth (i.e., all neurons in the culture having neurites). Alternatively, heterogeneity is the hallmark of simple nervous systems of identifiable neurons. It is possible that, in addition to individual differences in ionic currents and neuronal chemistry, different neurons may have differential responses to the $\mathrm{CF}$ present in our cultures.

Many of the features of neuritic outgrowth from isolated Helisoma neurons in vitro are reminiscent of previous observations on isolated vertebrate neurons. Rates of elongation of individual neurites within and among individual Helisoma neurons varied widely (6 to $40 \mu \mathrm{m}$ ) hr at $23^{\circ} \mathrm{C}$ ). Reported rates for various vertebrate neurons include: frog axons in culture, 15.6 to $56 \mu \mathrm{m} / \mathrm{hr}$ (Harrison, 1910); tadpole axons in vivo, $40 \mu \mathrm{m} / \mathrm{hr}$ (Speidel, 1941); frog at $26^{\circ} \mathrm{C}, 92 \mu \mathrm{m} / \mathrm{hr}$ (Lubinska and Olekiewicz, 1950); chick neurons, 7 to $51 \mu \mathrm{m} / \mathrm{hr}$ (Hughes, 1953); and regenerating mammalian axon, 83 to $124 \mu \mathrm{m} /$ hr (Ramón y Cajal, 1928). Growth in Helisoma neurons occurs at the tips of neurites by means of growth cones (Figs. 6 and 7). The general morphological characteristics of Helisoma growth cones (Fig. 7) are easily recognized as those described for most vertebrate growth cones. Membrane organelles include waving filopodia and flattened lamellapodia. Flattened regions of membrane comprise sites of axon-substrate apposition near the growth cone and are resorbed in axon segments more proximal to the cell body. Short time span (minutes) estimates of the rates of advance of growth cones can be widely variant. The "growth rate" of the leading edge can be high or the growth cone can actually retreat for short distances. This type of uneven, saltatory movement may be an essential feature for substrate "sampling" and directional growth (Letourneau, 1978; Gunderson and Barrett, 1979, 1980).

\section{Electrical excitability and growth}

All cells judged visually to be viable neurons (adhering, spherical, phase bright, yellow-pigmented cells) are electrically excitable regardless of culture condition or the expression of neuritic outgrowth. Quantitative measurements of electrical parameters have not been performed because the variation within experimental groups was similar to the variation among groups. A broad range of individual neuronal characteristics is observed; each neuron can be recognized as an individual by its electrical parameters. These individual characteristics appear stable on repeated penetrations over moderate periods of time ( $\sim 2$ to 3 days) in neurons whose morphologies are also stable over these time intervals (either spherical or with extensive neurites).

We did not record from individual neurons throughout the transition from spherical to neurite-bearing morphologies; therefore, we are presently unable to define further possible changes in the excitability accompanying neurite outgrowth. In systems of developing neurons, discrete changes in ionic events occur simultaneously with the elaboration of neuronal processes (reviewed by Spitzer, 1979). Recently, Meiri et al. (1981) described changes from $\mathrm{Ca}^{2+}$-dependent action potentials to $\mathrm{Na}^{+}$. dependent action potentials in regenerating cockroach giant axons. It is possible that our observation of only very short duration action potentials in neurite-bearing isolated Helisoma neurons may reflect similar changes in the ionic bases of excitability in these growing neurons. Further experimentation is required to assess this possibility.

\section{Properties of Helisoma conditioning factor}

Exogenous trophic agents are capable of acting either in solution directly or by associating with the substratum. Both soluble and surface-associated CFs may exist in Helisoma. At least one component of $\mathrm{CF}$ can be bound tightly to polylysine surfaces (as to Millipore filters) rather than acting as a soluble factor leached from the surface (Fig. 12, no activity on the control halves of the dishes). Although growth on adsorbed CF is qualitatively indistinguishable from growth on CMHL-15, soluble factors could exist. Regardless of the number of components in Helisoma CF, the quality of outgrowth from isolated neurons cultured with CF is comparable to that expressed by neurons regenerating within central ganglia cultured in vivo.

$\mathrm{CF}$ is present in conditioning brains, is released from the brains, and can promote neurite outgrowth by binding to the polylysine substratum. Neurite outgrowth in cultured intact ganglia initiates independently of exogenous CF, while outgrowth from isolated neurons requires 
$\mathrm{CF}$. The fact that $\mathrm{CF}$ is derived from brains raises another consideration: whether CF plays a role in situ. Endogenous $\mathrm{CF}$ within ganglia could promote neurite outgrowth and the added $\mathrm{CF}$ in organ culture may enhance outgrowth simply by raising the titer of CF within the ganglia. It is entirely possible that other factors, such as those found in tissue culture sera, could promote growth of Helisoma neurons. However, growth with the homospecific Helisoma CF, with its possible specificity compared to serum, may be less prone to the introduction of artifacts.

The ability to compare events in situ with the same events in cell culture could allow the examination of underlying mechanisms. Early attempts to culture isolated Helisoma neurons in defined media failed because neurons did not produce outgrowth-one of the critical features of neuronal plasticity. The behavior displayed by isolated Helisoma neurons in conditioned medium indicates that these neurons maintain the fundamental properties required for the iteration of many of the aspects of neuronal plasticity that we have observed previously in situ. The increase in experimental tractability afforded by cell culture can be coupled with the increased resolution provided by the use of identified neurons characteristic of this species.

\section{References}

Adler, R., and S. Varon (1980) Cholinergic neuronotrophic factors. V. Segregation of survival and neurite-promoting activities in heart-conditioned media. Brain Res. 188: 437448.

Banker, G. A. (1980) Trophic interactions between astroglial cells and hippocampal neurons in culture. Science 209: 809810.

Beiswanger, C. M., and J. W. Jacklet (1975) In vitro tests for a circadian rhythm in the electrical activity of a single neuron in Aplysia californica. J. Comp. Physiol. 103: 19-37.

Bray, D. (1973) Branching patterns of individual sympathetic neurons in culture. J. Cell Biol. 56: 702-712.

Bray, D. (1979) Mechanical tension produced by nerve cells in tissue culture. J. Cell Sci. 37: 391-410.

Bulloch, A. G. M., and S. B. Kater (1981) Selection of a novel connection by adult molluscan neurons. Science 212: 79-81.

Bulloch, A. G. M., S. B. Kater, and A. D. Murphy (1980) Connectivity changes in an isolated molluscan ganglion during in vivo culture. J. Neurobiol. 11: 531-546.

Chen, J. S., and R. Levi-Montalcini (1970) Long term cultures of dissociated nerve cells from the embryonic nervous system of the cockroach Periplaneta americana. Arch. Ital. Biol. 108: 503-537.

Collins, F. (1978a) Axon initiation by ciliary neurons in culture. Dev. Biol. 65: 50-59.

Collins, F. (1978b) Induction of neurite outgrowth by a conditioned medium factor bound to the culture substratum. Proc. Natl. Acad. Sci. U. S. A. 75: 5210-5213.

Collins, F. (1980) Neurite outgrowth induced by the substrate associated material from nonneuronal cells. Dev. Biol. 79: 247-252.

Eccles, J. C., B. Libet, and R. R. Young (1958) The behavior of chromatolyzed neurons studied by intracellular recording. J. Physiol. (Lond.) 143: 11-40.

Furshpan, E. J., P. R. MacLeish, P. H. O'Lague, and D. D. Potter (1976) Chemical transmission between rat sympathetic neurons and cardiac myocytes developing in microcul- tures: Evidence for cholinergic, adrenergic and dual-function neurons. Proc. Natl. Acad. Sci. U. S. A. 73: 4225-4229.

Gunderson, R. W., and J. N. Barrett (1979) Neuronal chemotaxis: Chick dorsal-root axons turn toward high concentrations of nerve growth factor. Science 206: 1079-1080.

Gunderson, R. W., and J. N. Rarrett (1980) Characterization of the turning response of dorsal root neurites toward nerve growth factor. J. Cell Biol. 87: 546-554.

Hadley, R. D., and S. B. Kater (1979) Characterization of identified neurons of Helisoma maintained in in vitro organ culture for the study of regeneration. Soc. Neurosci. Abstr. 5 : 627.

Harrison, R. G. (1910) The outgrowth of the nerve fiber as a mode of protoplasmic movement. J. Exp. Zool. 9: 787-846.

Hughes, A. F. (1953) The growth of embryonic neurites. A study on cultures of chick neural tissue. J. Anat. 87: 150-162.

Jacobson, M. (1978) Developmental Neurobiology, Ed. 2, Plenum Press, New York.

Kaczmarck, L. K., M. Finbow, J. P. Revel, and F. Strumwasser (1979) The morphology of coupling of Aplysia bag cells within the abdominal ganglion and in cell culture. J. Neurobiol. 10: 535-550.

Kandel, E. R. (1976) Cellular Basis of Behavior: An Introduction to Behavioral Neurobiology, Freeman, San Francisco.

Kater, S. B. (1974) Feeding in Helisoma trivolvis: The morphological and physiological bases of a fixed action pattern. Am. Zool. 14: 1017-1036.

Kater, S. B., and R. D. Hadley (1980) Visualization of living, intracellularly stained neurons using a Silicon Intensified Target camera. Soc. Neurosci. Abstr. 6: 686.

Kater, S. B., and C. R. S. Kaneko (1972) An endogenously bursting neuron in the gastropod mollusc, Helisoma trivolvis: Characterization of activity in vivo. J. Comp. Physiol. 79: 114.

Kater, S. B., and C. H. F. Rowell (1973) Integration of sensory and centrally programmed components in generation of cyclical feeding activity in Helisoma trivolvis. J. Neurophysiol. 36: $142-155$.

Kater, S. B., C. B. Heyer, and C. R. S. Kaneko (1975) Identifiable neurons and invertebrate behaviour. In International Review of Physiology. Series 1, Vol. 3: Neurophysiology, C. C. Hunt, ed., pp. 1-51, Butterworths, London.

Letourneau, P. C. (1975a) Possible roles for cell-to-substratum adhesion in neuronal morphogenesis. Dev. Biol. 44: 77-91.

Letourneau, P. C. (1975b) Cell-to-substratum adhesion and guidance of axonal elongation. Dev. Biol. 44: 92-101.

Letourneau, P. C. (1978) Chemotactic response of nerve fiber elongation to nerve growth factor. Dev. Biol. 66: 183-196.

Lubinska, L., and M. Olekiewicz (1950) The rate of regeneration of amphibian peripheral nerves at different temperatures. Acta. Biol. Exp. (Warsaw) 15: 125-145.

Meiri, H., M. E. Spira, and I. Parnas (1981) Membrane conductance and action potential of a regenerating axonal tip. Science 211: 709-712.

Murphy, A. D., and S. B. Kater (1978) Specific reinnervation of a target organ by a pair of identified molluscan neurons. Brain Res. 156: 322-328.

Murphy, A. D., and S. B. Kater (1980a) Sprouting and functional regeneration of an identified neuron in Helisoma. Brain Res. 186: 251-272.

Murphy, A. D., and S. B. Kater (1980b) Differential discrimination of appropriate pathways by regenerating identified neurons in Helisoma. J. Comp. Neurol. 190: 395-403.

Nicholls, J. G., B. Wallace, and M. Adal (1977) Regeneration of individual neurons in the nervous system of the leech. In Synapses, G. A. Cotrell and P. N. R. Usherwood, eds., pp. 249-263, Academic Press, New York. 
Pollack, E. D., and V. Liebig (1977) Differentiating limb tissue affects neurite growth in spinal cord cultures. Science 197: 899-900.

Ramón y Cajal, S. (1928) Degeneration and Regeneration of the Nervous System, R. M. May, translator (1959), Hafner, New York.

Ready, D. F., and J. G. Nicholls (1979) Identified neurons isolated from leech CNS make selective connections in culture. Nature 281: 67-69.

Reichart, G. F., and P. H. Patterson (1977) Neurotransmitter synthesis and uptake by isolated sympathetic neurons in microcultures. Nature 270: 147-151.

Speidel, C. C. (1941) Adjustments of nerve endings. Harvey Lect. 36: 126-158.

Spitzer, N. C. (1979) Ion channels in development. Annu. Rev. Neurosci. 2: $363-397$.

Stewart, W. W. (1978) Functional connections between cells as revealed by dye-coupling with a highly fluorescent naphthalimide tracer. Cell 14: 741-759. 